\title{
Wider Angst und Resignation - im Kopf liegen alle Heilmittel bereit
}

\section{Jürg Kesselring}

Prof. Dr. med., Mitglied FMH

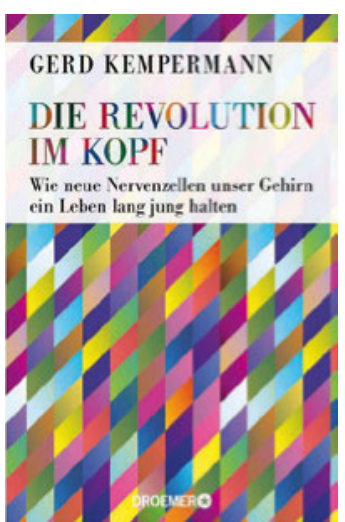

Gerd Kempermann

\section{Die Revolution \\ im Kopf}

Wie neue Nervenzellen unser Gehirn ein Leben lang jung halten 320 Seiten, 24.70 CHF. ISBN 978-3-426-27707-2 München: Droemer Verlag, 2016

In Zeiten, da höchste staatliche Autoritäten - die erklärtermassen nicht lesen - verkünden, dass man keine Fakten brauche, weil man die Wahrheit ja kenne, tut es besonders gut, ein Buch zu lesen, das solchen Behauptungen Substantielles entgegen zu halten vermag.

Wenn wir aktiv die Welt entdecken und in ihr handeln, wenn wir uns auf Neues einlassen, kurz: wenn wir lernen, dann zieht auch unser erwachsenes Gehirn dafür an entscheidender, strategischer Stelle neue Nervenzellen hinzu, die es bedarfsgerecht hervorbringt.

Gerd Kempermann aus Dresden ist seit Jahrzehnten ein anerkannter Forscher auf diesem Gebiet der Neuroregeneration, aber auch ein sehr umfassend gebildeter, sorgfältig formulierender und humorvoller Autor, dem man gerne durch die spannende Geschichte der adulten Neurogenese folgt. Neu an den in diesem Buch kenntnisreich beschriebenen Entwicklungen ist, dass bei der schon länger bekannten «Neuroplastizität» nicht nur stets neue Verbindungen zwischen den Nervenzellen (Synapsen) gebildet werden (und genutzt werden können/sollen), sondern tatsächlich auch neue Nervenzellen und zwar in jener für das Lernen und das Gedächtnis entscheidenden Region, dem Hippocampus. Er nennt sie so schön «das Tor zum Gedächtnis» - das Gedächtnis «sitzt» ja nicht einfach dort. Diese Neubildung ist ein Leben lang möglich und zwar in Abhängigkeit von unseren Aktivitäten und Erfahrungen. Dies führt dazu, dass jede und jeder von uns ein ureigenes, persönliches, individuelles Gehirn entwickelt. Es bleibe dahingestellt, ob dieses der Sitz oder einfach eine Bedingung sei für unsere erinnerte Autobiographie, für unsere Urteile und Vorhersagen, unsere Bildung und unsere emotionalen Wertungen, d.h. für unser Bewusstsein und unsere individuelle Persönlichkeit in der Wechselwirkung mit der natürlichen und sozialen Umwelt.

Diese adulten Neurogenese wurde und wird an verschiedensten biologischen Systemen untersucht, zum Beispiel an Kanarienvögeln, welche neue Lieder für ihren Brauttanz lernen und dabei neue Nervenzellen bilden (nach der Balz allerdings wieder vergessen) oder an Ratten und Mäusen in raffinierten Versuchsanordnungen. Dabei hat es sich seit Kempermanns eigenen bahnbrechenden Untersuchungen im anregenden Labor von Fred Gage am Salk Institut in La Jolla an der amerikanischen Westküste bewährt, die Tiere in einer reichhaltigen Umgebung sich entwickeln und handeln $\mathrm{zu}$ lassen und dann das Gehirn zu untersuchen. Denn dieses sieht strukturell völlig anders aus als dasjenige von (auch genetisch identischen) Tieren, die in einer sterilen und öden, anregungsfreien Umgebung aufgewachsen sind. Es gibt keinen begründeten Zweifel, dass ähnliches ebenso für uns Menschen gilt, wobei freilich «anregungsreiche Umgebung» nicht irgendeinen mate-

\section{Sehr wohltuend und anregend ist,} wie Kempermann gewisse «Gehirnmythen» darstellt und in ihre Schranken verweist.

riellen Reichtum meint, sondern eher die Möglichkeit, sich in einer Welt bewegen zu dürfen, in der man selber Probleme lösen kann. Es zeigt sich, dass die adulte Neurogenese nicht einfach nur ein reiner Reservemechanismus ist, sondern Teil eines fundamentalen Prinzips, das dem Lernen und unseren Gedächtnisleistungen zu Grunde liegt.

Die an Wissenschaftsgeschichte interessierten Leserinnen und Leser werden mit Interesse (und einigem 
Schmunzeln) die Auseinandersetzungen verschiedener Schulen begleiten, z.B. die legendäre der beiden Nobelpreisträger Cajal vs. Golgi zum «Keine-neuenNervenzellen-Mythos»: dieser beruht auf einem immer wieder unvollständig kolportierten Zitat («Im erwachsenen Gehirn sind die Nervenbahnen fixiert und unveränderlich - alles kann sterben, aber nichts kann regenerieren»), hatte doch selbst der zitierte Cajal so schön poetisch beschrieben: «[Die Hirnrinde] ... ist wie ein Garten voller unzähliger Bäume ..., die dank einer intelligenten Züchtung ihre Fortsätze vervielfachen können, ihre Wurzeln tief versenden, um immer mehr unterschiedliche Blüten und Früchte hervorzubringen" (Seite 80). Man wird auch die Faszination angewandter Techniken nicht übersehen können, z.B. wenn etwa in Turnhallen füllenden Apparaten einzelne $14 \mathrm{C}$ Atome aus Milliarden normaler Kohlenstoffatome herausgefischt werden, um sich zu Nutze zu machen, dass bis in die sechziger Jahre überirdische Atombombentests erfolgt sind, welche die Konzentration des Kohlenstoff Isotops $14 \mathrm{C}$ in der Atmosphäre haben ansteigen lassen und man nun auf

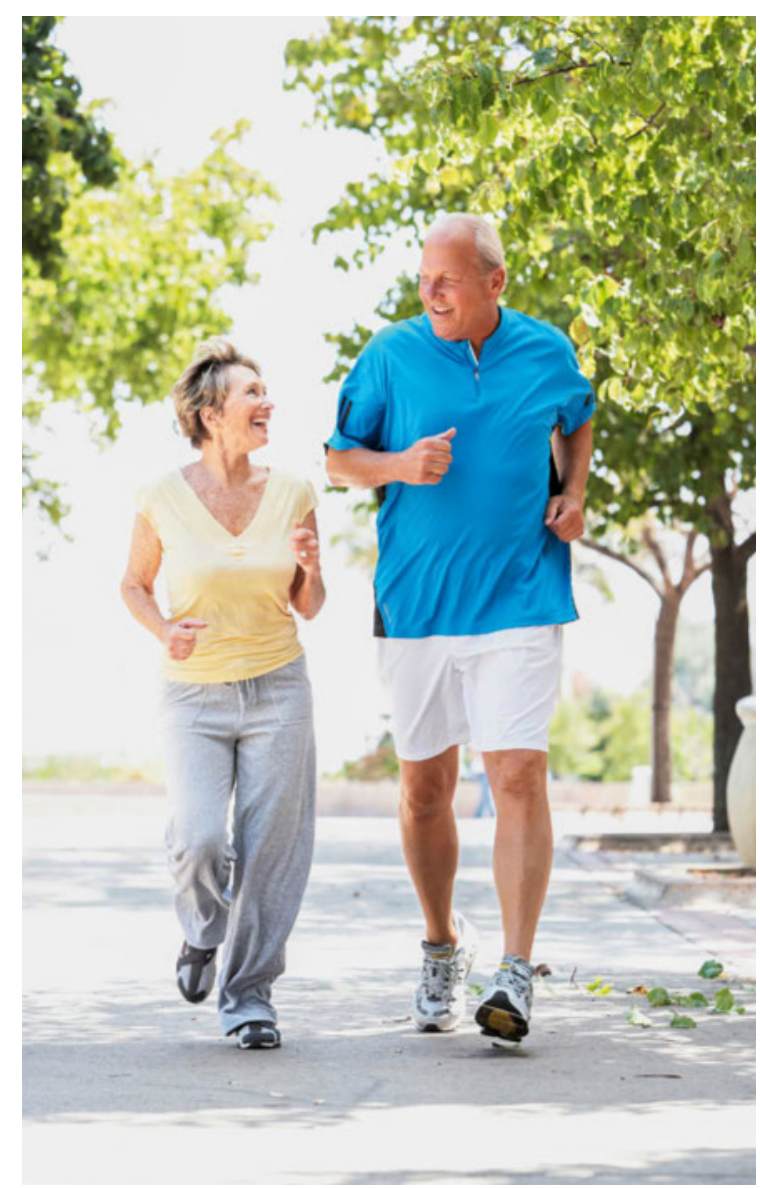

Sport und Bewegung allgemein steigert die Neurogenese. Kann man also der Demenz davonlaufen? diese Weise einen tatsächlichen Beweis dafür findet, dass neue Nervenzellen auch beim erwachsenen Menschen gebildet werden können. Schön ist auch der Dank an seinen Lehrer Fred H. Gage, dem er entscheidende Eigenschaften eines wirklich guten Wissenschaftlers attestiert: die «Seitwärtsbewegungen» - wie er als Wissenschaftler gelegentlich abseits der geraden Wege geht und offen ist für Entwicklungen auf anderen Feldern und damit eine Methode vorlebt, wie man auch allfälligen eigenen Kreativitäts- und Identifikati-

\section{Es führen verschiedene Kanäle zum Gehirn (die Sinnesorgane), es gibt aber nur einen Ausweg: die Motorik, d.h. Bewegung.}

onskrisen vorbeugen kann. Es wird sogar festgehalten, dass der Häretikerstatus gesund sei, aber anstrengend. Er freut sich an der selbstbewussten Bescheidenheit seiner Lehrer und erlebte sie als grosse Ermöglicher. Er beschreibt auch aus eigener Erfahrung den "glücklichen Zufall [Serendipity], die Mischung aus Weisheit und Glück, durch die etwas nicht ganz durch Zufall entdeckt wird».

Sehr wohltuend und anregend ist, wie Kempermann gewisse «Gehirnmythen» darstellt und in ihre Schranken verweist, zum Beispiel, dass wir angeblich nur 10\% unseres Gehirns nutzen (wie will man dies denn untersuchen können?) oder auch den Behaviorismus, der mit seinen simplen Reiz-Reaktionsschemen so lange Zeit die Psychologische Forschung dominiert hat. Zwischen Input und Output liegen ganze Welten, die wir erst sehr langsam zu ermessen beginnen, von ihrem Verstehen ganz zu schweigen. Die Gleichsetzung von biologischen Voraussetzungen und Mechanismen mit einem deterministischen, naturalistischen Weltbild ist zwar keineswegs zwingend und bei genauerer Betrachtung kontraproduktiv, aber es herrschte damals (und auch noch heute in gewissen Kreisen) die Angst vor, dass zu viel Biologie die Macht der Pädagogik bzw. Politik mindern könnte. Kempermann scheut sich nicht, in so heiss umstrittenen gesellschaftlichen und politischen Kontroversen wie der «Stammzellendebatte» klar und differenziert Stellung zu beziehen.

Die an Neuroanatomie Interessierten kommen in den detaillierten Beschreibungen der Hippocampus-Region auf die Rechnung, besonders wenn sie die sorgfältig ausgewählt Abbildungen mit den treffenden Beschreibungen im farbigen Bildteil konsultieren. Oder bei der «subventrikulären Zone» (SVZ), jener Nische in der Ventrikelwand, aus der sich die gesamte Hirnrinde entwickelt, sodass man verstehen kann, dass die adulte 
Neurogenese hier eine Fortsetzung der Neurogenese während der embryonalen Entwicklung ist.

Schon einer der ersten Wissenschaftler, der Synapsenfunktionen untersuchte, Donald Hebb, machte die Beobachtung, dass seine Labortiere sich ganz anders verhielten, nachdem er sie über das Wochenende nach Hause genommen hatte und seine Kinder mit ihnen spielten. Interessant - und für FitnessstudioBetreiber vielleicht nützlich zu wissen - ist die Beobachtung, dass Versuchstiere, die sich in freier Umgebung bewegen durften (wie üblich fast $12 \mathrm{~km}$ pro Nacht ...), auch dann gerne das Laufrad bevorzugten, selbst wenn es in der freien Natur stand. Und da zeigte sich, dass sowohl körperliche Aktivität ebenso wie eine reizreiche Umgebung die adulten Neurogenesen im Hippocampus steigern. Es führen verschiedene Kanäle zum Gehirn (die Sinnesorgane), es gibt aber nur einen Ausweg: die Motorik, d.h. Bewegung. Diese Eingangsund Ausgangsseiten sind sehr eng miteinander verknüpft, z.T. über rasch wirksame Kurzschlussverbin-

\section{Das Gehirn kann aufgefasst werden als ein Speicher plus die Fähigkeit zur Vorhersage.}

dungen. Nun zeigen verschiedenste Untersuchungen, dass körperliche Aktivität die kognitiven Funktionen verändern kann, «Bewegung ist gut für das Gehirn». Empfohlen wird also ganz praktisch ein reservebildender Lebensstil.

Prof. Dr. med.

Jürg Kesselring

Chefarzt Neurologie und Neurorehabilitation

Rehabilitationszentrum

Kliniken Valens

Juerg.Kesselring[at]

kliniken-valens.ch plus die Fähigkeit zur Vorhersage, denn wenn wir lernen, können wir etwas in der Zukunft besser und müssen nicht die gleichen Fehler zweimal machen. Aus verschiedenen Experimenten und Beobachtungen lässt sich feststellen, dass das Erlernen eines Kontextes Hippocampus-abhängig ist. Die neuen Nervenzellen können also Informationen mit einer Art Zeitstempel versehen. Sie dienen wahrscheinlich dazu, eine Erinnerung und Vorstellung flexibel auf den neuesten Stand zu bringen. Für das Lernen an sich braucht man die neuen Zellen wahrscheinlich nicht, sie verhelfen

\section{Die neuen Nervenzellen können also Infor-} mationen mit einer Art Zeitstempel versehen.

aber, neue Informationen flexiblen in bestehende Kontexte $\mathrm{zu}$ integrieren und erlauben eine Anpassung an Neuartigkeit und Komplexität und erhöhen damit die Adaptationsfähigkeit. Dies ist ohne Zweifel bei den schweren neurologischen Erkrankungen, die mit einem Abbau der Nervenzellen einhergehen (z. B. Alzheimer-Krankheit), von grösster, sicher auch prophylaktische Bedeutung. Man könnte «der Demenz davonlaufen». Es wird geschätzt (und erhofft - S. 250), dass durch modifizierbare Risikofaktoren (Blutdruck und Übergewicht im mittleren Lebensalter, Depression, Rauchen, niedriger Bildungsstand und körperliche Inaktivität) etwa ein Drittel der Alzheimer-Demenz ursächlich erklärt werden kann. Lernen und Sport (und aktives Musizieren), Bewegung allgemein und bei uns Menschen damit auch die Zahl (und sicher auch die Art) der sozialen Kontakte steigern die Neurogenese, Depressionen vermindern sie.

Das ganze, spannend geschriebene Buch ist eine wohl fundierte Empfehlung und Anregung, ein aktives Leben zu führen!
Bildnachweis

(c) Monkeybusinessimages | Dreamstime.com 